of infantile gastroenteritis throughout the world. It is important to note, however, that to date few specimens have been examined from regions where gastroenteritis mortality rates are particularly high. The use of the electron microscope for rapid and reliable detection of rotaviruses in clinical specimens and the development of serological methods of identification will greatly facilitate further studies.

\section{Does Hex A depend on Hex B?}

\section{from a Correspondent}

THE question of the relationship of the $\mathrm{N}$-acetyl hexosaminidases is still in dispute. It has been known for six years or so that two major electrophoretic forms of hexosaminidase (A and $B$ ) occur in human tissues and also that one of these (Hex $A$ ) is deficient in patients with Tay-Sachs disease, a fatal recessive disorder of infants characterised by the accumulation of a complex lipid, the ganglioside $\mathbf{G M}_{2}$, the in vivo substrate of the enzyme, in the brain. The deficiency of both enzymes is also found in some patients -a variant which is often called Sandhoff's disease. It has generally been thought to be unlikely that two separate and independent gene loci code for the hexosaminidases, as the deficiency of both A and B in Sandhoff's disease would presumably require two separate mutations (at the locus for $A$ and for $B$ ). The deficiency of Hex B alone has never been reported and TaySachs disease is extremely rare-thus the coincidence of the two homozygous mutant genes in the same individuals would be expected to be very rare indeed. But twenty or so cases have in fact already been reported.

Biochemical and immunological evidence suggests that the enzymes are closely related, and two types of model have been proposed for their relationship. One is that Hex $\mathbf{A}$ and $\mathbf{B}$ possess similar and dissimilar subunits (for example $\mathrm{A}=\alpha \beta ; \mathrm{B}=\beta \beta$ ). The other is that $\mathbf{A}$ and $\mathbf{B}$ are essentially the same protein, one enzyme being a secondarily (possibly enzymatically) modified form of the other.

In theory one might expect to learn something from the study of somatic cell hybrids. Human-rodent hybrid cells progressively lose their human chromosomes. By analysing the hybrids in a search for the coincident presence or absence of human enzymes and chromosomes genes can be assigned to particular chromosomes. When more than one gene (situated on more than one chromosome) is required to code for a particular protein or proteins the number of independently located genes involved can be determined.

Information of this kind has been obtained for hexosaminidase but the results from different laboratories are conflioting (van Someren et al., Humangenetik, 18, 171, 1974; Lalley et al., Proc. natn. Acad. Sci. U.S.A., 71, 1569, 1974; Nguyen Van Cong et al., C.r. Acad. Sci. Paris., 278, 1761, 1974; Gilbert et al., Proc. natn. Acad. Sci. U.S.A., 72, 263, 1975). In the most recent paper on the subject Gilbert et al. claim that there are indeed two independent gene loci that code for the hexosaminidases. The gene for Hex $B$ is assigned to chromosome 5 and the gene for Hex A (in agreement with the other groups) is shown to be on the same chromosome as mannose phosphate isomerase(MPI), which has now been assigned to chromosome 15 (van Heyningen et al., Ann. Hum. Genet., 38, 295; 1975). This result differs from that obtained previously by Lalley et al. and Nguyen Van Cong et al. These authors never observed the retention of human Hex A in the absence of Hex B in the hybrid cells and suggested that Hex A requires the presence of two human genes for its expression and B requires only one of the two genes (which now seems to be on chromosome 5). These findings were consistent with both the favoured models for Hex A/Hex B relationship. The lack of observation of one particular combination is not however in itself very convincing evidence for a dependence model. The best supporting evidence would come from clones which have MPI but neither Hex A nor Hex B, predicted from this model for those clones which have chromosome 15 but not 5. Very few definite clones of this type have so far been reported. Gilbert et al. on the other hand claim to have clones of all the possible types (including Hex $A+$, Hex $B-$ ), which are consistent with their model. There are, however, some major problems that cast doubt on the interpretation of their results.

In order to avoid implicating two mutations in Sandhoff's disease, Gilbert et al. have suggested that the defect may be a mutation in a locus which controls the expression of both $A$ and $B$ or makes an activator. The first problem is that there is some evidence that certain Sandhoff patients at least make non-active antigenically cross reactive 'hexosaminidase' proteins (Srivastava and Beutler, J. biol. Chem., 249, 2054, 1974; Carroll and Robinson, Biochem. $J ., 137,217,1974)$. If the control model were right then no such protein should be synthesised. Second, another informative hexosaminidase variant has been reported in a healthy parent of a patient with Sandhoff's disease (Dreyfus et al., New Eng. J. Med., 292, 61,
1975). This individual has a deficiency of both Hex A and Hex B, as detected using an artificial substrate, and is probably heterozygous for the Sandhoff allele and one which produces kinetically abnormal $\mathbf{A}$ and $\mathbf{B}$ enzymes (which seem to hydrolyse the natural but not the artificial substrates for the enzyme). This variant strongly suggests that the enzymes must have polypeptides in common.

From the technical point of view, other workers have commented on difficulties involved in identification of the human hexosaminidase components in hybrid cells, and it may be that a problem of this kind is leading to misclassification of the enzymes.

So, the argument goes on ... .

\section{Genes, enzymes and behaviour}

from T. J. Crow

THAT heredity is a powerful determinant of behaviour was first cogently argued in Lange's book Crime as Destiny (Allen and Unwin, London, 1931). The controversy about the genetic contribution to intelligence has recently had a lively renaissance; $H$. J. Eysenck, a protagonist of the innate factor school, has also championed the view that there are important hereditary influences on the personality variables neuroticism and extraversion. In the more limited field of overt psychiatric disease the contribution of genetic factors to the major psychoses, schizophrenia and manicdepressive illness, is now generally accepted and has recently been reemphasised by studies on adopted offspring of schizophrenic patients (for example Heston, Science, 167, 249; 1970; Kety, Am. J. Psychiat., 131, 957; 1974). Evidence for genetic factors in neurotic illness (for example Shields, Monozygotic Twins; Oxford University Press, 1962) is less well-known, but perhaps equally significant.

Attempts to study the links between genes and behaviour have led to the development of animal models. In selective breeding experiments in rats Broadhurst (Nature, 184, 1517; 1959) was able to demonstrate a genetic influence determining 'emotionality', as defined by increased defaecation rates in an open field apparatus, and an independent factor of "extroversionintroversion' (J. exp. Psychol., 56, 349; 1958).

Bovet and his colleagues (Science, 163, 139; 1969) demonstrated very striking differences between strains, and consistencies within strain, in the rates, and temporal patterns, of aquisition of a conditioned avoidance response in mice. Such experiments invite specula- 